Reprod. Nutr. Dévelop., 1985, 25 (6), 1017-1028.

\title{
Dosage radioimmunologique de l'ACTH chez le lapin : relations stress - ACTH - corticostéroïdes et diarrhées
}

\author{
M. MONNIER, B. DESBALS (1) \\ avec la collaboration technique de J. C. FAGE et O. NARDOU
}

Laboratoire de Physiologie animale, Faculté des Sciences, 123, rue A. Thomas 87060, Limoges Cedex, France.

Summary. ACTH radioimmunoassay in the rabbit : relationships between plasma ACTH response to stress, corticosteroids and diarrhea.

Plasma ACTH was measured in young and adult rabbits with a CEA-SORIN kit. The reliability of the analysis was controlled by liquid chromatography, several biochemical tests such as parallelism and reproducibility and several physiological tests such as plasma ACTH response to ethylic ether stress, to adrenalectomy, to dexamethasone and metopyrone.

In young diarrhoeic rabbit plasma, ACTH $(293 \pm 45 \mathrm{pg} / \mathrm{ml})$ and corticosteroid $(111 \pm 13 \mathrm{ng} / \mathrm{ml})$ levels were significantly much higher than in young healthy rabbits (ACTH : $130 \pm 35 \mathrm{pg} / \mathrm{ml}$; corticosteroids : $66 \pm 7 \mathrm{ng} / \mathrm{ml}$ ).

Furthermore, transporting young rabbits by car caused such violent stress that plasma ACTH levels increased from $52 \pm 11$ to $130 \pm 35 \mathrm{pg} / \mathrm{ml}$.

A causal relationship between stress - ACTH and diarrhoea is discussed.

\section{Introduction.}

La corticotropine que l'on considère à l'heure actuelle comme étant une des hormones clés liée au stress a donné lieu à de nombreuses recherches chez le rat, I'homme, ainsi que chez quelques autres espèces animales. Son dosage est principalement réalisé à l'aide d'une technique radioimmunologique dérivée de la méthode originale du dosage de l'insuline, mise au point par Yalow et Berson en 1960. Depuis le premier dosage de l'ACTH utilisable, réalisé par Yalow et al. en 1964, de nombreuses variantes en ont été proposées ne différant que par des détails techniques. Un des problèmes majeurs posé par tous les dosages radioimmunologiques des hormones polypeptidiques réside dans la nature de l'anticorps contenu dans l'immunsérum, tant en ce qui concerne l'affinité de cet anticorps vis-à-vis de l'antigène que dans l'adéquation entre l'activité biologique et la réactivité immunologique de ce que I'on dose (ORTH, 1974).

(1) Demandes de tirés à part à B. DESBALS. 
Nous intéressant au problème posé par la diarrhée chez le lapereau d'élevage industriel, nous avons été amené à doser l'ACTH plasmatique, notre hypothèse étant que cet état pathologique pourrait être la conséquence d'une situation de stress. Pour cela nous avons utilisé une trousse commercialisée par le CEA SORIN (référence ACTHK) qui permet de doser I'ACTH directement dans le plasma humain sans pratiquer d'extraction préalable, bien que celle-ci soit recommandée par certains auteurs (Schöneshöfer et al., 1981).

A notre connaissance, le dosage de l'ACTH n'ayant pas été réalisé à l'aide de ce kit chez cet animal, d'une façon systématique, nous avons donc voulu tout d'abord nous assurer que la technique proposée pouvait s'avérer utilisable et répondait au but que nous nous proposions; c'est pourquoi nous avons réalisé dans un premier temps un certain nombre d'essais dont nous exposons cidessous les résultats. Ceci nous a permis ensuite d'aborder le problème de la relation pouvant exister entre la diarrhée chez le lapereau et le taux d'ACTH plasmatique. En effet dans les jours qui suivent le sevrage qui a lieu aux environs du $28^{\mathrm{e}}$ jour après la naissance, le laperéau peut présenter des diarrhées souvent mortelles qui déciment les élevages. Le sevrage pourrait représenter un stress dont les effets endocriniens induiraient ces diarrhées. Une éventuelle relation causale stress ACTH et diarrhée a donc été recherchée.

D'autre part la corticotropine contrôlant la production des hormones corticostéroïdes, principalement le cortisol et la corticostérone, par les glandes corticosurrénales nous avons parallèlement dosés les corticostéroïdes totaux.

\section{Matériel et méthodes.}

1. Les animaux. - Nous avons utilisé des lapins "Solam-Solaf " soit adultes mâles âgés de 10 à 12 semaines, soit des lapereaux âgés de 5 à 7 semaines ; chez le lapereau la difficulté de la reconnaissance du sexe conduisant à une contention génératrice de stress nous ne l'avons pas systématiquement recherchée ; toutefois afin de vérifier s'il y avait ou non une différence liée au sexe nous avons déterminé celui-ci après sacrifice d'un lot d'animaux (le lot III de la figure 5 , 17 animaux sains). Nous n'avons pas observé de différence liée au sexe tant en ce qui concerne les taux plasmatiques de l'ACTH que des corticostéroïdes.

2. - Les prélèvements de sang. - Chez l'adulte, le sang est prélevé entre 16 h 30 et 17 h 30 afin d'éviter d'introduire une variation liée au nycthémère.

Ce prélèvement est effectué à la veine marginale de l'oreille, récupéré sur EDTA (1 mg p. $\mathrm{ml}$ ) puis centrifugé à $9800 \mathrm{~g}$ pendant $2 \mathrm{~min}$ (Eppendorf 3200 ). Le plasma séparé est immédiatement congelé et conservé à $-30^{\circ} \mathrm{C}$. Dans le cas où les échantillons sont conservés pendant une période excédant une semaine on ajoute au moment de la prise de sang un inhibiteur enzymatique (Aprotinin protease inhibitor, SIGMA Chem.) à la concentration de $100 \mathrm{UIK} / \mathrm{ml}$ de sang. Chez le lapereau le sang est prélevé en moins d'une minute par ponction dans la veine cave inférieure après sacrifice de l'animal par élongation rapide de la moelle épinière cervicale; nous avons aussi effectué des prélèvements par décapitation. 


\section{3. - Le dosage de l'ACTH : description technique}

a) Le tampon : L'incubation des échantillons est réalisée dans un milieu constitué de tampon véronal $0,02 \mathrm{M}$ à pH 8,4 contenant du mercaptoéthanol à $0,2 \%$ destiné à réduire la dégradation de l'ACTH en protégeant de l'oxydation la méthionine qui se trouve en position 4 à partir de l'extrémité $\mathrm{N}$-terminale. Ce tampon est en outre utilisé pour dissoudre l'hormone radioactive et l'anticorps présentés sous forme lyophilisée ; il servira également à préparer la suspension de charbon destinée à séparer les formes liées et libres de l'hormone en fin d'incubation.

b) L'ACTH marquée à l'iode 125 (émetteur $\gamma$ ) est d'origine porcine, son activité spécifique est de l'ordre de $300 \mu \mathrm{Ci} / \mu \mathrm{g}$, elle est présentée sous forme lyophilisée en présence d'albumine humaine et d'un inhibiteur enzymatique.

c) L'ACTH standard est de I'ACTH synthétique humaine (1-39) lyophilisée et comprenant du plasma humain dépourvu d'ACTH ; au moment de l'emploi on la solubilise dans l'eau distillée. La gamme standard est généralement comprise entre $30 \mathrm{pg} / \mathrm{ml}$ et $1000 \mathrm{pg} / \mathrm{ml}$; elle comprend en outre un standard $O$ qui contient seulement du plasma humain dépourvu d'ACTH. Au moment de l'emploi on reconstitue les standards à l'aide d'eau distillée.

d) L'immunsérum $22 \mathrm{~B}$ a été obtenu chez le lapin après injection d'ACTH porcine couplée à de l'albumine bovine (Vague et al., 1971).

e) Le charbon est du charbon végétal activé (Norit) mis en suspension extemporanément dans le tampon véronal et maintenu tout au long de sa distribution sous agitation magnétique dans un bain à $+4^{\circ} \mathrm{C}$. Chaque tube reçoit l'équivalent de $10 \mathrm{mg}$ de charbon sauf ceux destinés à mesurer l'activité totale.

4. - Le dosage de l'ACTH : réalisation technique. - Le dosage a été réalisé dans des tubes en polystyrène $(75 \times 12 \mathrm{~mm})$ placés dans un bain froid maintenu à $+4^{\circ} \mathrm{C}$ selon le protocole indiqué dans le tableau 1 .

TABLEAU 1

Protocole de pipettage du dosage R.I.A. de la corticotropine

\begin{tabular}{|c|c|c|c|c|}
\hline Essais & $\begin{array}{l}\text { Tampon } \\
(\mathrm{ml})\end{array}$ & $\begin{array}{c}\text { Standard ou échantillon } \\
\mathrm{ml}\end{array}$ & $\underset{(\mathrm{ml})}{\mathrm{ACTH}^{125}}$ & Immunsérum \\
\hline Activité totale $T$ & 1,3 & & 0,1 & \\
\hline $\begin{array}{l}\text { Activité non spécifique (liée aux } \\
\text { standards) } A_{s}\end{array}$ & 0,8 & 0,1 (standard 0 ) & 0,1 & \\
\hline $\begin{array}{l}\text { Point zéro de la courbe } 0 \\
\text { (détermination de la capacité de liaison } \\
\text { antigène anticorps) }\end{array}$ & 0,7 & 0,1 (standard 0 ) & 0,1 & 0,1 \\
\hline Standard $n^{\circ} 1$ à $n^{\circ} 5$ & 0,7 & 0,1 & 0,1 & 0,1 \\
\hline Echantillon à doser & 0,7 & 0,1 & 0,1 & 0,1 \\
\hline $\begin{array}{l}\text { Activité non spécifique (liée aux } \\
\text { échantillons) } A_{E}\end{array}$ & 0,8 & 0,1 & 0,1 & \\
\hline
\end{tabular}

Tous les essais sont en triple exemplaire, sauf les échantillons qui sont en double avec pour chacun d'eux un essai « activité non spécifique » $A_{E}$. 
$\mathrm{L}^{\prime}$ incubation dure $48 \mathrm{~h}$ à $+4^{\circ} \mathrm{C}$, la séparation fraction libre-fraction liée de I'hormone se fait également à froid, par addition à tous les tubes sauf ceux destinés à la mesure de l'activité totale de $0,5 \mathrm{ml}$ de suspension de charbon dans un délai aussi bref que possible, elle est suivie d'une agitation de quelques secondes au Vortex puis d'une centrifugation à froid à $2000 \mathrm{~g}$ d'une durée de $15 \mathrm{~min}$. Le surnageant contenant la fraction liée radioactive est transféré dans des tubes de comptage dont la radioactivité $\gamma$ est mesurée pendant 1 min au compteur Packard prias auto-gamma.

5. - Le dosage des corticostérö̈des totaux. - II est réalisé selon la technique décrite par Murphy et al. (1963) modifiée par Leclerq et al. (1969), adaptée par Bobet (1978) c'est un dosage par radiocompétition.

a) Principe du dosage : Du cortisol tritié (1,2 ${ }^{3} \mathrm{H}$ Cortisol Amersham) et les corticostéroïdes présents soit dans des échantillons standards soit dans les échantillons de plasma entrent en compétition vis-à-vis des sites de fixation présents sur une $\alpha$-glycoprotéine plasmatique (Transcortine ou CBG - corticosteroïd binding globulin -1. La quantité d'hormone tritiée fixée est fonction de la quantité d'hormone froide présente dans le milieu. On établit une courbe étalon (fig. 1). Les quantités inconnues sont déterminées par comparaison avec cette courbe.

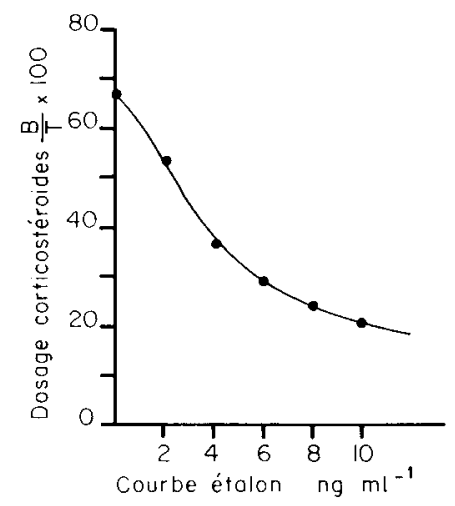

FIG. 1. - Exemple de courbe étalon pour le dosage des corticostérö̈des.

b) Réalisation technique : De la CBG est préparée à partir de plasma humain épuré par adsorption des corticostéroïdes sur du charbon à $39^{\circ} \mathrm{C}$ pendant $30 \mathrm{~min}$. On réalise un complexe $\mathrm{CBG} /{ }^{*} \mathrm{~F}(\mathrm{~F}=$ cortisol selon la classification de Kendall)

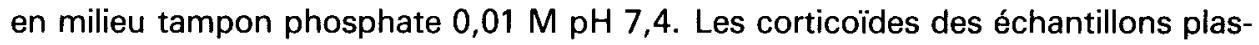
matiques sont extraits par du dichloro-méthane (Merck et après évaporation sous courant d'azote les échantillons sont repris dans un volume adéquat d'éthanol absolu (Merk) ; le dosage est réalisé sur une fraction aliquote de cette solution. Les aliquots et les échantillons alcooliques des standards sont amenés à sec, on ajoute alors une quantité identique pour tous les échantillons de complexe $\mathrm{CBG} /{ }^{*} \mathrm{~F}$ puis on place les tubes dans un bain-marie à $40^{\circ} \mathrm{C}$ pendant $45 \mathrm{~min}$ ce qui a pour effet de dissocier le complexe. Suit une incubation de $2 \mathrm{~h}$ à $+4{ }^{\circ} \mathrm{C}$ qui 
permet au complexe de se reformer ; c'est à ce moment qu'a lieu la compétition entre les corticostéroïdes froids et le cortisol tritié.

On sépare les hormones libres des hormones liées par adsorption sur charbon ; finalement par une centrifugation poussée on récupère le surnageant qui contient le complexe dont on mesure la fraction radioactive (émission $\beta$ ).

\section{Résultats.}

\section{I. - Etude qualitative et quantitative du dosage de l'ACTH chez le lapin.}

Différents essais biologiques et techniques ont eu pour objet d'évaluer la qualité de cette technique; ainsi chez le lapin la sécrétion d'ACTH sera soit stimulée (stress à l'éther éthylique, surrénalectomie, traitement à la métopirone) soit déprimée (traitement à la dexaméthasone); par ailleurs la reconnaissance de la validité des mesures sera estimée par des analyses chromatographiques, courbes de parallélisme et tests de reproductibilité inter et intra-dosages. Enfin l'ACTH plasmatique de lapins adultes mâles normaux sera évaluée.

1. - Etude de la courbe standard du dosage de l'ACTH. - La courbe étalon moyenne à partir de 11 essais est représentée sur la figure 2. Nous avons utilisé la représentation en $B / B_{0} \times 100$ en coordonnées linéaires; $B$ (Bound) représentant la fraction d'hormone marquée à l'iode 125 liée à l'anticorps après incubation en présence d'hormone froide, $B_{0}$ étant la fraction liée à l'anticorps en l'absence d'ACTH froide. La capacité de liaison $\left(B_{0} / T \times 100\right)$ mesurée sur 11 essais est de $51 \% \pm 2 \%$ (SEM) avec un coefficient de variation de $14 \%$; l'activité non spécifique As mesurée sur 11 essais est de $11 \% \pm 0,6 \%$ avec un coefficient de variation de $16 \%$ (fig. 2 ).

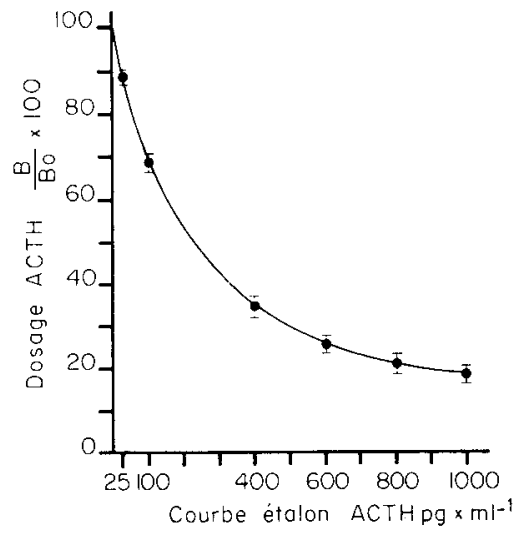

FIG. 2. - Courbe étalon moyenne pour le dosage de l'ACTH.

2. - Chromatographie d'un échantillon de plasma provenant d'un animal ayant subi un stress à l'éther : dosage radioimmunologique des fractions susceptibles de contenir de l'ACTH (fig. 3). - 1,5 $\mathrm{ml}$ de ce plasma est déposé sur une colonne de Séphadex G 50 Fine (Farmacia Fine Chemi.) ; l'élution est réalisée à 
$4{ }^{\circ} \mathrm{C}$, par un tampon phosphate à $\mathrm{pH} 7,4$ contenant $0,3 \%$ de sérum-albumine bovine ; le débit d'élution est de $19,2 \mathrm{ml} / \mathrm{h}$. Les fractions susceptibles de contenir I'ACTH qui sera dosé par RIA sont identifiées par comparaison avec la chromatographie préalable identique d'ACTH ${ }^{125}$ en solution dans du plasma de lapin dépourvu d'ACTH, celle-ci ayant été extraite du plasma par du charbon végétal Norit : $500 \mathrm{mg}$ pour $10 \mathrm{ml}$ de plasma.

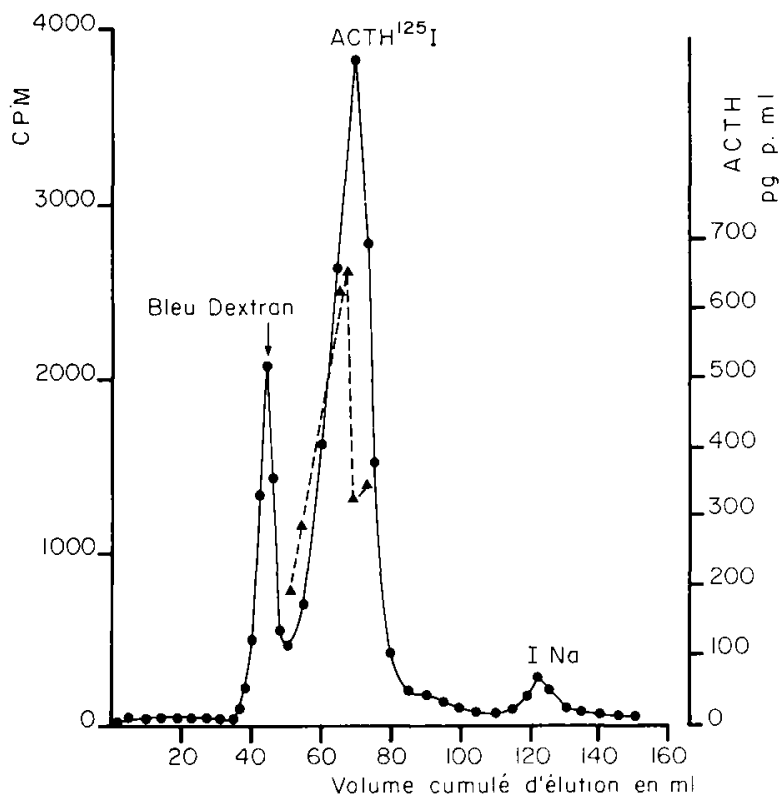

FIG. 3. - Identification de I'ACTH plasmatique chez le lapin.

Chromatographie de plasma sur colonne $(1 \mathrm{~m} \times 16 \mathrm{~mm})$. Séphadex $\mathrm{G} 50$ Fine. Elution par du tampon phosphate $\mathrm{pH} 7,4$ albuminé à $0,3 \%$ par de la sérum-albumine bovine. Débit $19,2 \mathrm{ml} / \mathrm{h}$.

3. - Test de parallélisme (fig. 4). - Il consiste à doser un même échantillon plasmatique à plusieurs dilutions réalisées avec du plasma dépourvu d'ACTH afin de vérifier que le dosage est indépendant des quantités mesurées, ainsi que d'éventuelles substances, polypeptides ou protéines présentes dans le plasma. Nos résultats sur plusieurs échantillons sont satisfaisants.

4. - Reproductibilité du dosage (tableau 2). - Nous avons vérifier la repro-

TABLEAU 2

\begin{tabular}{lcc}
\hline \multirow{2}{*}{ Date du dosage } & \multicolumn{2}{c}{ ACTH $(\mathrm{pg} / \mathrm{ml})$} \\
\cline { 2 - 3 } & Nombre d'essais & moyenne \pm SEM \\
\hline 200684 & 20 & $427 \pm 7$ \\
270684 & 16 & $430 \pm 11$ \\
121084 & 16 & $480 \pm 9,5$
\end{tabular}

La comparaison lot par lot à l'aide du test $t$ de Student ne fait pas apparaître de différence significative au seuil de $5 \%$. 


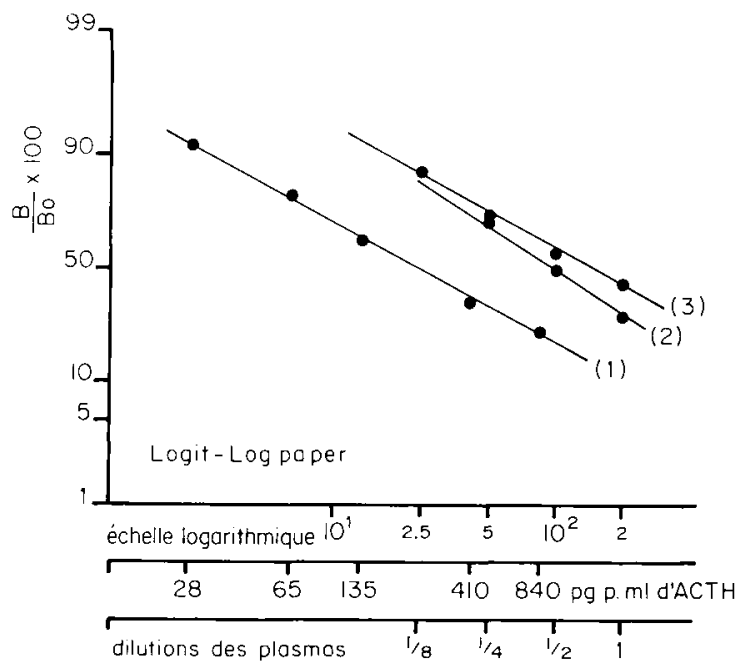

FIG. 4. - Courbes de parallélisme.

La courbe de parallélisme est réalisée en reportant sur le papier logit-log les valeurs B/Bo $\times 100$ en ordonnées et en faisant subir à l'axe des abcisses une translation ce qui permet en reportant sur celui-ci les diverses dilutions effectuées à partir d'un échantillon quelconque de dégager une droite qui doit être parallèle à la courbe étalon. En faisant subir à la droite ainsi obtenue une translation selon l'axe des abcisses (ici vers la gauche) celle-ci doit venir recouvrir la courbe étalon. On aurait pu également obtenir le même type de courbe en faisant subir une translation à l'axe des ordonnées.

courbe (1): courbe étalon,

courbe (2) : courbe obtenue à partir de plasma d'animal stressé,

courbe (3) : courbe obtenue à partir du même plasma dilué aú $1 / 2$ avec du plasma dépourvu d'ACTH.

ductibilité intra et inter dosage en introduisant un même échantillon que nous avons dosé 52 fois à différents moments.

5. - Dosage de l'ACTH chez des lapins adultes normaux. - L'ACTH a été dosé sur un total de 20 animaux adultes présents au laboratoire ; leur taux moyen est de $280 \pm 30 \mathrm{pg} \mathrm{p}$. $\mathrm{ml}$ (moyenne \pm ESM).

6. - Effet d'un stress à l'éther éthylique. - L'éther éthylique provoque un stress d'installation rapide entraînant une forte augmentation du taux d'ACTH plasmatique (Anderson E., 1966 ; Hary, Dupouy et Chatelain, 1981 ; Khan et al., 1984). L'application d'éther éthylique conduit à un évanouissement en 2 à 3 min ; $10 \mathrm{~min}$ après, le taux d'ACTH est de $667 \pm 60 \mathrm{pg} / \mathrm{ml}$ (moyenne \pm ESM sur 9 animaux) pour des valeurs initiales de $261 \pm 26 \mathrm{pg} / \mathrm{ml}$ (moyenne \pm ESM sur 9 animaux).

7. - Effet de la surrénalectomie. - Les animaux sont surrénalectomisés (Desbals, 1967) à $17 \mathrm{~h}$ et sacrifiés le lendemain vers $8 \mathrm{~h}$, soit $15 \mathrm{~h}$ après l'opération. Le taux plasmatique d'ACTH est passé de $173 \pm 32 \mathrm{pg} / \mathrm{ml}$ à $1225 \pm 148$ (moyenne \pm ESM).

8. - Effet de la dexaméthasone. - La dexaméthasone est réputée comme bloqueur de l'axe hypothalamo-hypophyso-surrénalien (Usategui et al., 1976 ; De 
Souza et Van Loon 1982). $2,5 \mathrm{mg} / \mathrm{kg}$ i.v. de dectancyl Roussel (acétate de dexaméthasone) sont administrés à 4 animaux et le taux de l'ACTH est évalué en fonction du temps. L'effet inhibiteur est spectaculaire. En effet le taux moyen d'ACTH avant l'injection de dectancyl qui est de $221 \pm 121 \mathrm{pg} / \mathrm{ml}$ est ramené à 0 dans les $4 \mathrm{~h}$ suivant l'injection et demeure tel au-delà de $33 \mathrm{~h}$, l'inhibition est donc totale et durable.

9. - Effet de la métopirone (tabl. 3). - La métopirone ou métyrapone CibaGeigy ( $250 \mathrm{mg}$ i. m. par animal) inhibe la $11 \beta$-hydroxylation et de ce fait bloque la production des 11-hydroxycorticostéroïdes (Chart et al., 1958 ; Metcalf et Beaven, 1968) ce qui lève leur rétro-contrôle négatif sur la sécrétion d'ACTH.

TABLEAU 3

Effets de la métopirone

ACTH plasmatique en $\mathrm{pg} \times \mathrm{ml}^{-1}$

Expérimentation $\left(^{*}\right) \mathrm{n}^{\circ}$

$\mathrm{t}_{0}=$ taux témoins

$t_{1}=t_{0}+30 \mathrm{~min}$

$t_{2}=t_{0}+60 \min$

$t_{3}=t_{0}+120 \mathrm{~min}$

$t_{4}=t_{0}+240 \mathrm{~min}$

$t_{5}=t_{0}+360 \mathrm{~min}$

1
220
260

métopirone $250 \mathrm{mg}$ i.m. par animal

920

760

1100

1400

280

La levée du frein corticosurrénalien exalte la sécrétion d’ACTH.

(*) 1 animal par expérimentation.

\section{II. - Diarrhées, ACTH et corticostéroïdes.}

1. - Nous avons sacrifié des lapereaux apparemment sains au laboratoire, dès leur arrivée après un transport routier depuis l'élevage industriel $(30 \mathrm{~km}, 60$ $\mathrm{min}$ ). Trois séries d'animaux (49 lapereaux au total) ont été réalisées : le taux moyen d'ACTH plasmatique est de $130 \pm 18 \mathrm{pg} / \mathrm{ml}$; le taux moyen des corticostéroïdes de $66 \pm 7,4 \mathrm{ng} / \mathrm{ml}$ (fig. 5 ).

2. - Des lapereaux apparemment sains ont été sacrifiés directement chez les éleveurs avec prélèvement soit par la veine cave inférieure (15 cas) soit après décapitation (12 cas). II n'y a pas de différence statistiquement significative entre les taux d'ACTH à partir de ces deux modes de prélèvements sanguins, et le taux moyen général pour les 27 animaux est de $46 \pm 7 \mathrm{pg} / \mathrm{ml}$; par contre les taux de corticostéroïdes sont de $46 \pm 11 \mathrm{ng} / \mathrm{ml}$ dans le premier lot (V.C.I.) et de 13,5 \pm $4 \mathrm{ng} / \mathrm{ml}$ pour l'autre lot (décapitation). Ces deux lots sont eux significativement différents (fig. 5 ).

II apparaît que les taux d'ACTH mesurés au laboratoire après le transport routier sont nettement plus élevés, ce qui signifie que ce transport constitue une agression notable pour les lapereaux; ceci est corroboré quoique d'une façon 
moins nette par le taux des corticostéroïdes (fig. 5). Par ailleurs le mode de prélèvement sanguin peut expliquer la différence observée avec les taux de corticostéroïdes obtenus lors des sacrifices chez l'éleveur ; cette différence tient probablement à la proximité anatomique des surrénales lors du prélèvement par la veine cave inférieure. Cette opinion est renforcée par le fait que I'ACTH, elle, ne varie pas en fonction du mode de prélèvement, ce qui indique une absence d'effet de stress du fait de la rapidité d'exécution. Nous avons donc, pour des raisons pratiques prélevé le sang par la veine cave inférieure, les surrénales n'étant pas en tout état de cause stimulées puisque le taux d'ACTH ne varie pas.

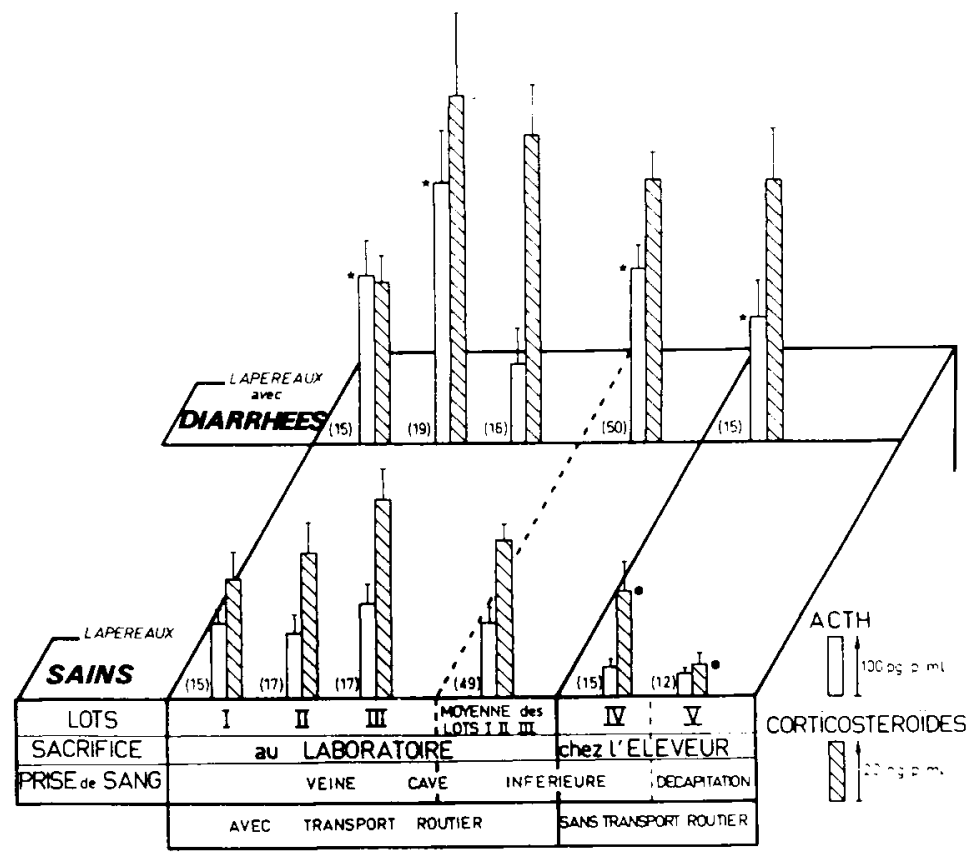

FIG. 5. - Stress, ACTH, corticostéroïdes et diarrhées chez le lapereau.

Les lapereaux atteints de diarrhées présentent des taux plasmatiques d'ACTH et de corticostéroïdes très nettement supérieurs à ceux des lapereaux sains.

1) Nombre d'animaux.

Valeurs movennes \pm SEM.

- : taux significativement différents selon le mode de prélèvement du sang ; $\checkmark$ et $\star$ : taux significativement différents entre lapereaux sains et malades.

3. - Animaux atteints de diarrhées. - L'expérimentation a porté sur 65 lapereaux. Trois lots de 15, 19 et 16 animaux furent transportés jusqu'au laboratoire pour $y$ être sacrifiés et un lot de 15 animaux fut sacrifié directement chez l'éleveur. Pour les lots de 15 et 19 lapereaux sacrifiés au laboratoire ainsi que pour le lot sacrifié chez l'éleveur les taux d'ACTH sont très significativement plus élevés que chez les animaux apparemment sains (fig. 5). Dans le lot des 16 animaux le taux d'ACTH est comparable à celui des animaux sains (figure 5 ). Néanmoins si I'on compare le taux moyen du groupe " animaux malades " dans sa totalité $293 \pm 45 \mathrm{pg} / \mathrm{ml}$ avec celui des « animaux sains » $130,4 \pm 18,0 \mathrm{pg} / \mathrm{ml}$, la diffé- 
rence est hautement significative au seuil de $1 \%$ (par le calcul de l'écart réduit). La différence est également significative dans le cas des animaux sacrifiés directement chez l'éleveur : "lapins sains » $52 \pm 11 \mathrm{pg} / \mathrm{ml}$, "lapins malades " $214 \pm$ $62 \mathrm{pg} / \mathrm{ml}$ (fig. 5). Enfin la comparaison des corticostérö̈des entre les " animaux sains " $66 \pm 7,4 \mathrm{ng} / \mathrm{ml}$ et les " animaux malades " $111 \pm 13 \mathrm{ng} / \mathrm{ml}$ va dans le même sens ; différence significative à $1 \%$ en utilisant le même type de comparaison.

D'autre part dans le cas du lot de 16 lapereaux où le taux moyen de l'ACTH ne diffère pas de celui des " animaux sains " (16 cas) les corticostéroïdes révélateurs de l'activation corticotrope hypothalamo-hypophysaire sont pour leur part significativement plus élevés (117 $\pm 21 \mathrm{ng} / \mathrm{ml})$ que chez les normaux (fig. 5).

\section{Discussion.}

1. - Le dosage de l'ACTH plasmatique que nous avons développé chez le lapin fournit des résultats cohérents en accord avec ce que l'on trouve dans la littérature quant aux désordres endocriniens induits par le stress à l'éther, la surrénalectomie, l'effet de la dexaméthasone ainsi que celui de la métopirone. Le rétrocontrôle négatif exercé par la cortico-surrénale envers l'hypophyse chez le lapin est très sensible. Ainsi la dexaméthasone a un effet inhibiteur d'installation rapide ; cet effet est puissant et durable. Il est permis de penser au vu des résultats obtenus à la suite de l'action de la métapirone que cette rétroaction négative doit être permanente à l'état normal chez le lapin puisque l'interruption momentanée de la sécrétion des corticostéroïdes par cette drogue induit dès 30 min après son injection une élévation importante de I'ACTH plasmatique ; néanmoins cet effet est relativement fugace puisque $4 \mathrm{~h}$ plus tard les taux d'ACTH sont redevenus normaux. La surrénalectomie pour sa part levant définitivement le frein cortico-surrénalien conduit à des élévations persistantes et importantes de I'ACTH plasmatique. Ainsi il apparaît que chez le lapin une altération de la sécrétion des corticostéroïdes induit une réponse immédiate et ample de la sécrétion hypophysaire d'ACTH.

D'autre part, I'identification chromatographique suivie du dosage RIA de l'ACTH dans des fractions d'un plasma de lapin stressé séparées sur colonne de Séphadex nous permet d'affirmer qu'il y a une bonne corrélation entre le corps que l'on dose et la sécrétion biologique. Néanmoins ceci n'exclut pas l'existence de différentes formes d'ACTH telles que l'ont rapporté Orth et Nicholson (1977) ou Yalow et Berson (1971). Enfin au plan analytique les courbes de parallélisme bien qu'imparfaites sont satisfaisantes ainsi que les essais de reproductibilité intra et inter dosage.

2. - La comparaison globale des taux d'ACTH et de corticostéroïdes entre les animaux sains (49) et diarrhéiques (50) fait ressortir des différences très significatives. Toutefois ces différences sont parfois moins marquées au niveau des couples de lots sains-malades de même origine (élevage - date de prélèvement) ; ceci tient vraisemblablement au fait que les animaux sont prélevés au hasard chez l'éleveur sur les seuls critères d'âge et d'apparence saine ou malade (diarrhée) ; 
par suite nous ignorions au moment du prélèvement si tel lapin apparemment sain, le resterait ou si tel animal malade était à un stade d'évolution plus ou moins tragique de la diarrhée. D'autre part l'observation des résultats ne nous permet pas de constater une relation quantitativement étroite entre le taux d'ACTH et celui des corticostéroïdes correspondants. Toutefois chez les animaux malades, globalement, l'augmentation des corticostéroïdes suit celle de I'ACTH. Cependant dans le cas du lot $n^{\circ}$ III (fig. 5, 16 animaux malades et 17 animaux sains) les taux respectifs d'ACTH ne sont pas statistiquement différents entre sains et malades, mais on observe des taux plasmatiques de corticostéroides significativement plus élevés chez les malades. Ceci peut s'expliquer par le fait que la production et l'excrétion de I'ACTH par l'hypophyse est intermittente et que d'autre part sa vitesse de disparition est relativement rapide alors que la réponse des surrénales à la stimulation corticotrope est relativement plus lente à s'installer et que, surtout, la durée de vie des corticostéroïdes plasmatiques est beaucoup plus longue (De Souza et Van Loon, 1982). Enfin il existe une différence hautement significative entre le taux d'ACTH des animaux sains sacrifiés au laboratoire après transport et celui des animaux sacrifiés directement chez l'éleveur ; ceci est incontestablement à mettre au compte du transport qui entraîne des perturbations physiologiques constatées par ailleurs chez d'autres espèces (Dantzer et Mormède, 1979).

\section{Conclusion.}

Le Kit ACTHK du CEA-SORIN peut être utilisé d'une façon satisfaisante pour effectuer le dosage de la corticotropine chez le lapin.

Nous avons observé des différences significatives des taux d'ACTH et de corticostéroïdes totaux entre les animaux sains et les animaux malades atteints de diarrhées. Une éventuelle relation causale Stress-ACTH ou corticostéroïdes et diarrhées peut ètre recherchée. Enfin le transport constitue indubitablement un stress pour le lapin.

Reçu en mars 1985.

Accepté en juillet 1985 .

\section{Références}

ANDERSON E., 1966. Adrenocorticotrophin-releasing hormon in peripheral blood : increase during stress. Science, 152, 379-380.

BOBET J. P., 1978. Variations saisonnières du métabolisme périphérique de la testostérone et du cortisol chez le Hérisson (Erinaceus europaeus L.). Th. Doct. 3ème Cycle, Univ. Poitiers.

CHART J. J., SHEPPART H., ALLEN M. J., BENCZE W. L., GAUNT R., 1958 . New amphenone analogs as adrenocorticol inhibitors. Experientia, 14, 151.

DANTZER R., MORMĖDE P., 1979. Le stress en élevage intensif. Actualités scientifiques et agronomiques de l'INRA, Masson Paris.

DESBALS B., 1967. Rôles respectifs des hormones surrénaliennes et hypophysaires dans le déterminisme des troubles entraînés par la surrénalectomie chez le lapin. Effets extrasurrénaliens de la corticotropine. Th. Doct. ès Sci. Toulouse. 
DE SOUZA B., VAN LOON R., 1982. Stress induced inhibition of the plasma corticosterone response to a subsequent stress in rats : a non adrenocorticotropin mediated mechanism. Endocrinology, 110, 23-33.

HARY L., DUPOUY J. P., CHATELAIN A., 1981. Pituitary response to bilateral adrenalectomy, metyrapone treatment and ether stress in the newborn rats. Biol. Neonate, 39, 28-36.

KHAN M. N., MIREL R. D., ONTJES D. A., GHOSH A. P., LAZARUS L. H., DI AUGUSTINE R. P., 1984. Adrenocorticotropin radioimmunoassay: properties of antisera against synthetic ACTH (1-24) and its clinical application. Hormone. Res., 20, 129-137.

LECLERCQ R., COPINSCHI C., FRANCKSON I. R. M., 1969. Dosage par compétition du cortisol plasmatique. Rev. fran. Etude clin. biol., 14, 815-819.

METCALF, M. G., BEAVEN D. W., 1968. The metopirone test of pituitary corticotropin release. Evaluation of 101 tests. Amer. J. Med. 45, 176-186.

MURPHY B. E. P., ENGELBERT W., PATTE C. J., 1963. Simple method for the determination of plasma corticoïds. J. clin. Endoc., 23, 293-300.

ORTH D. N., 1974. Adrenocorticotropic hormone and melanocyte stimulatin hormone (ACTH and MSH), 125-160. In methods of hormone radioimmunoassay JAFFEE B. M., BEHRMAN H. R., Acad. Press, New York.

ORTH D. N., NICHOLSON N. E., 1977. Different molecular forms of ACTH. Ann. N.Y. Acad. Sci, 297, 27-48.

SCHÖNESHÖFER M., SCHEFZIG B., FENNER A., WEBER B., DULCE H. J., 1981. Evaluation of a sensitive radioimmunoassay of plasma corticotropin using commercial reagents. J. clin. Chem. clin Biochem., 19, 75-79.

USATEGUI R., OLIVER C., VAUDRY H., LOMBARDI G., ROZENBERG I., MOURRE A. M., 1976. Immunoreactive $\alpha \mathrm{MSH}$ and ACTH level in rat plasma and pituitary. Endocrinology, 98, 189-196.

VAGUE P., OLIVER C., JAQUET P., VAGUE J., 1971. Le dosage radioimmunologique de I'ACTH plasmatique. Résultats chez les sujets normaux. Rev. europ. Etudes clin. biol., 16, 485-493.

YALOW R. S., BERSON S. A., 1960. Immunoassay of endogenous plasma insulin in man. J. clin. Investig., 39, 1157-1175.

YALOW R. S., BERSON S. A., 1971. Size heterogeneity of immunoreactive human ACTH in plasma and in extracts of pituitary glands and ACTH producing thymona. Biochem. biophys. Res. Commun., 44, 439-445.

YALOW R. S., GLICK S. M., ROTH J., BERSON S. A., 1964. Radioimmunoassay of human plasma ACTH. J. clin. Endocrinol. Metab., 24, 1219-1225. 